\title{
Edge Location and Identification Method for Electronic Components based on Improved Canny Algorithm
}

\author{
Yuan Chao, Fan Shi, Wentao Shan, Dong Liang \\ School of Mechanical Engineering, Jiangsu University of Technology, Changzhou 213001, Jiangsu, \\ China \\ Received: June 22, 2021. Revised: November 30, 2021. Accepted: January 8, 2022. Published: January 10, 2022.
}

\begin{abstract}
The position identification of SMD electronic components mainly uses Canny edge detection algorithm to detect the edges of specific elements, benefited from its computational simplicity. The traditional Canny algorithm lacks the adaptability in gradient calculation and double thresholds selection, which may affect the location and identification accuracy of specific elements in electronic components. In this paper, an improved canny edge detection algorithm is proposed. The gradient magnitude is calculated in four directions, i.e., horizontal, vertical, and diagonal. Both the high and low thresholds can be adaptively determined based on the grayscale distribution information, to increase the adaptability of edge identification. The experimental results show that the proposed method can better locate the true edges of specific elements in electronic components with a reasonable processing speed, compared with the traditional Canny algorithm, and has been successfully applied on practical real-time vision inspection on SMD electronic components.
\end{abstract}

Keywords-gradient calculation; electronic component; Canny algorithm; threshold; edge location

\section{INTRODUCTION}

$\mathrm{W}$ ITH the rapid development of electronic science theory and technology, the demands of miniaturization and integration in electronic systems have been growing rapidly [1], and electronic components have been developed into small size, light weight and thin surface mounting components, other than traditional plug-in components [2]. Surface mount technology (SMT) is used to identify the marking points of the printed circuit board (PCB) and the components to be mounted by positioning device [3]. Automatic placement of components on the predetermined position of circuit board and the corresponding installation work with high efficiency and accuracy can then be finished. The most intuitive evaluations of an SMT production line are basically the installation speed, and the mounting accuracy. Quality issues of electronic circuit products have consistently been arousing people's attention. Machine vision based measurement and inspection system is the critical process in the manufacturing and quality testing of electronic circuit products, which completes the works of image acquiring, image processing and then the target parameters and key quality information extracting [4]. In the actual production, external environmental factors and internal processing issues may lead to damage to a certain extent on the surface or the package of electronic components, which will seriously affect their operational stability and durability. Therefore, the position identification and defect detection of electronic components with high efficiency play an important role in vision inspection, in which accurate edge location and identification of specific elements in electronic components is the premise.

At present, many scholars have done research works on electronic components edge detection and positioning via machine vision. There are two main kinds of methods of locating circuit board components based on image processing. The first one is template matching based on known templates of the components. Xiao et.al [5] proposed a method for circuit board components positioning and testing based on speed-up robust features. The position was found through the coordinated relation of the matching points, using the seven optimized invariant moment features method. However, this method suffered from the matching speed and could hardly applied on real-time vision inspection. The other method is based on the extracted key characteristics of the components to perform positioning and identification. Zhou et.al [6] proposed an improved Harris corner detection algorithm to extract target contours of quad flat no-lead (QFN) chips and solve the image tilt issue of QFN images during vision inspection and defect detection. Considering the influence of the image's brightness on positioning, a method combining phase congruence with Hough circle was proposed by Chen et.al [7]. This method has shown preferable anti-interference capability, but limitations exist in devices with high precision. Jiang et.al [8] used the teaching method to eliminate the first PCB board positioning error, and then the PCB board fiducial mark was used via shape identification and coordinate calculation, to eliminate the remaining PCBs positioning and manufacturing errors, and ensure the repeat mounting accuracy of the SMT machine. In [9], visual defects in PCBs can be simply detected by dissecting and pointing out the differences on the output images using a 
method of image subtraction and blob detection based on OPENCV. Chao et.al [10] proposed a multilevel image segmentation algorithm for QFN chips, to locate the lead and central heat sink regions, and reveal simultaneously the defect regions, i.e., scratch, resin bleed and stain. Nevertheless, the heavy computational burden of multilevel image segmentation still remains a potential problem.

Vision based location and identification methods for PCB and its surface mounted devices (SMD) components, such as QFN, SOP, et.al, mainly include template matching, regional growth, morphological processing, and edge detection [11-14], among which Canny edge detection algorithm is the most widely used in real-time application, benefited from its computational simplicity. However, due to the sensitivity to the external environment and various types of non-linear noises during the electronic components image processing procedure, the traditional Canny algorithm lacks the adaptability in gradient calculation and double thresholds selection, which may affect the location and identification accuracy of specific elements in electronic components. In order to achieve better denoising, more smooth and accurate transition to the edge image of the electronic components, an improved Canny algorithm is proposed in this paper. The remainder of this paper is as follows. The second section mainly discusses the traditional Canny algorithm, and an improved Canny algorithm with gradient magnitude calculation in 4 directions and adaptive thresholds selection is proposed in the third section. The experimental results and discussions are presented in section 4 . The conclusions are made in the last section.

\section{TRADITIONAL CANNY EDGE DETECTION ALGORITHM}

\section{A. Canny Edge Detection Criteria}

Canny edge detection operator follows the three performance criteria for judging the optimal edge [15]. (1) Signal-to-noise ratio (SNR) criterion. The accuracy of detected true edge points by the algorithm increases with the increase of SNR. (2) Positioning accuracy criterion. The detected edge points should be as close as possible to the true edge points. (3) Single edge response criterion. The probability of one single edge generating multiple responses should be as low as possible to suppress the response of false edges.

\section{B. Gaussian Filter and Gradient Calculation}

The set of pixels with the most drastic changes in the gradient direction of the image is defined as the image edge, so the edge of the image can be determined by calculating the local maximum of the image gradient, which is calculated by the first derivative of the two-dimensional Gaussian filter $h(x, y, \sigma)$, as shown in $\mathrm{Eq}(1)$. The original image $f(x, y)$ is then convolved with Gaussian function to obtain the smoothed image $g(x, y)$, as in $\mathrm{Eq}(2)$.

$$
\begin{gathered}
h(x, y, \sigma)=\frac{1}{2 \pi \sigma^{2}} \exp \left(-\frac{x^{2}+y^{2}}{2 \sigma^{2}}\right) \\
g(x, y)=h(x, y, \sigma)^{*} f(x, y)
\end{gathered}
$$

where $\sigma$ denotes the standard deviation of Gaussian function, and $(x, y)$ is the coordinate of pixel in $X$ axis and $Y$ axis in the image.

The gradient of the smoothed image $g(x, y)$ can be obtained by the first-order differential operator in $X$ axis and $Y$ axis directions in $2 \times 2$ neighborhood, shown as $\mathrm{Eq}(3)$ and $\mathrm{Eq}(4)$,

$$
\begin{aligned}
& g_{x}{ }^{\prime}(x, y)=G_{x}=[g(x+1, y)-g(x, y) \\
& +g(x+1, y+1)-g(x, y+1)] / 2 \\
& g_{y}{ }^{\prime}(x, y)=G_{y}=[g(x, y+1)-g(x, y) \\
& +g(x+1, y+1)-g(x+1, y)] / 2
\end{aligned}
$$

where $G_{x}, G_{y}$ denote the approximate values of the first-order differential of the smoothed image $g(x, y)$ in $X$ axis and $Y$ axis directions, respectively. The gradient magnitude $M(x, y)$ and the gradient direction $\theta(x, y)$ can be calculated as $\mathrm{Eq}(5)$ and $\mathrm{Eq}(6)$, respectively.

$$
\begin{gathered}
M(x, y)=\sqrt{G_{x}^{2}+G_{y}^{2}} \\
\theta(x, y)=\arctan \left(G_{y} / G_{x}\right)
\end{gathered}
$$

Finally, the non-maximum suppression of local pixels is performed and then the edge points are judged by double thresholding method.

\section{Canny Algorithm Flow}

The traditional Canny algorithm flow is composed of the following specific steps:

Step 1. The image is converted to grayscale and the Gaussian filter is used for image smoothing to eliminate the noise in the grayscale image and smooth the edge contours.

Step 2. The gradient magnitude and direction of the smoothed image are calculated.

Step 3. Non-maximum suppression method is performed in four directions, i.e., horizontal, vertical, and diagonal, based on the image gradient magnitude. The local maximum points along the gradient direction are preserved, and the others are suppressed.

Step 4. The local maximum points in this step will be further judged whether they are true edge points, by the double thresholding detection. The double thresholds, i.e., the high and the low thresholds are commonly chosen artificially with fixed parameters. The local maximum points are compared with the chosen double thresholds and only the points that satisfy the detection criteria are identified as true edge points and then the connectivity analysis is performed to link edges.

\section{IMPROVED CANNY EDGE DETECTION ALGORITHM}

\section{A. Disadvantages of Traditional Canny Algorithm}

The traditional canny algorithm is superior to other general edge detection algorithms in practical vision-based measurement and inspection applications. However, disadvantages and deficiencies also exist as follows. 
(1) The smoothing parameter $\sigma$ in Gaussian filter is selected artificially, and the size of filter window tends to affect the smoothing effect. It is hard for Gaussian filter to balance edge preserving and noise smoothing, especially non-linear noise.

(2) First-order partial derivative finite difference in the $2 \times 2$ neighborhood is applied to calculate the gradient magnitude and direction [15], which is sensitive to the noise, and easy to ignore some true edge details.

(3) The double thresholding detection method needs manual selection of the high and low thresholds, which is non-adaptive and will affect the accuracy of true edges judgement and false edges removing.

In view of the above disadvantages, an improved gradient calculation method and an adaptive threshold selection method are proposed.

\section{B. Improved Gradient Calculation Method}

In order to better suppress the non-linear noise and preserve edge information in electronic components images captured by industrial cameras in actual vision inspection, median filter is introduced to perform image smoothing before gradient calculation, as shown in $\operatorname{Eq}(7)$,

$$
g(x, y)=\operatorname{median}\{f(x-k, y-l),(k, l \in W)\}
$$

where $f(x, y)$ and $g(x, y)$ are the original image and the smoothed image, respectively, and $W$ is the neighborhood window with the size of $3 \times 3$.

The original $2 \times 2$ neighborhood first-order differential operator used in gradient calculation is replaced by $3 \times 3$ neighborhood, 8-connectivity, and 4 directions, i.e. horizontal, vertical, and diagonal, inspired from the conventional Sobel operator. The gradient calculation of the smoothed image $g(x, y)$ can be improved as Eq.(8) (11),

$$
\begin{aligned}
& g_{x}^{\prime}(x, y)=G_{x}=[g(x-1, y+1)+2 \cdot g(x, y+1)+g(x+1, y+1) \\
& -g(x-1, y-1)-2 \cdot g(x, y-1)-g(x+1, y-1)] \\
& g_{y}^{\prime}(x, y)=G_{y}=[g(x-1, y-1)+2 \cdot g(x-1, y)+g(x-1, y+1) \\
& -g(x+1, y-1)-2 \cdot g(x+1, y)-g(x+1, y+1)] \\
& g_{45}{ }^{\prime}(x, y)=G_{45}=[g(x-1, y)+2 \cdot g(x-1, y+1)+g(x, y+1) \\
& -g(x, y-1)-2 \cdot g(x+1, y-1)-g(x+1, y)] \\
& g_{135}{ }^{\prime}(x, y)=G_{135}=[g(x, y+1)+2 \cdot g(x+1, y+1)+g(x+1, y) \\
& -g(x, y-1)-2 \cdot g(x-1, y-1)-g(x-1, y)]
\end{aligned}
$$

where $G_{x}, G_{y}, G_{45}, G_{135}$ denote the approximate values of the first-order differential of the smoothed image $g(x, y)$ in 4 corresponding directions, respectively.

Then the gradient magnitude $M(x, y)$ and the gradient direction $\theta(x, y)$ can be obtained combining the four directions, as $\mathrm{Eq}(12)$ and $\mathrm{Eq}(13)$, respectively.

$$
\begin{gathered}
M(x, y)=\sqrt{G_{x}^{2}+G_{y}^{2}+G_{45}^{2}+G_{135}^{2}} \\
\theta(x, y)=\arctan \left(G_{y}^{2} / G_{x}^{2}\right)
\end{gathered}
$$

\section{Adaptive Threshold Selection Method}

Thresholding is a simple and effective technique that widely used in image segmentation and edge detection, to separate objects from the background. According to the difference of gray value of the target and the background regions in image, as the difference becomes more significant, the variance between the two classes becomes larger. The threshold is determined by minimizing the variance inside the class, or equivalently, by maximizing the variance between the classes. Since the images of electronic components are commonly and simply composed of the specific elements as lead, heat sink, and plastic encapsulated package [16], the aforementioned threshold can be used as a clear distinction between the lead, heat sink regions and the package regions.

The aforementioned global threshold $T$ is defined to separate the image into the target class with the pixel range of $[0, T]$ and the background class with the pixel range of $[T+1, L-1]$, where $L$ denotes the gray level. The number of pixel value $i$ is defined as $n_{i}$, and the total pixels number can be calculated as $N=\sum_{i=0}^{L-1} n_{i}$. The probability $p_{i}$ of the occurrence of pixel value $i$ can be defined as Eq.(14).

$$
p_{i}=\frac{n_{i}}{N}
$$

The sum of variance of the target class and the background class can be defined as Eq.(15),

$$
\sigma_{B}^{2}(T)=\omega_{1}(T)\left(\mu_{1}(T)-\mu_{\mathrm{M}}\right)^{2}+\omega_{2}(T)\left(\mu_{2}(T)-\mu_{\mathrm{M}}\right)^{2}
$$

where $\mu_{\mathrm{M}}$ denotes the mean intensity of the input grayscale image, $\mu_{1}$ and $\mu_{2}$ denote the mean levels of the two classes, $\omega_{1}$ and $\omega_{2}$ denote the probability of the two classes. The abovementioned variables satisfy the equation as Eq.(16).

$$
\left\{\begin{array}{l}
\mu_{\mathrm{M}}=\sum_{i=0}^{L-1} i p_{i} \\
\omega_{1}(T)=\sum_{i=0}^{T} p_{i} \\
\omega_{2}(T)=\sum_{i=T+1}^{L-1} p_{i} \\
\omega_{1}(T)+\omega_{2}(T)=1 \\
\mu_{1}(T)=\sum_{i=0}^{T} i p_{i} / \omega_{1}(T) \\
\mu_{2}(T)=\sum_{i=T+1}^{L-1} i p_{i} / \omega_{2}(T)
\end{array}\right.
$$

Finally, the optimal threshold $T^{*}$ can be configured by maximizing the objective function using Eq.(17), and then set as the high threshold in the double thresholding detection method. The low threshold can be determined by multiplying the high threshold with a constant coefficient, which is set to 0.5 to get a relatively ideal thresholding detection result. In 
such a way, the Canny algorithm can determine the appropriate double thresholds adaptively, depending on the input image.

$$
T^{*}=\arg \max _{0 \leq T \leq L-1}\left\{\sigma_{B}^{2}(T)\right\}
$$

\section{Improved Canny Algorithm Flow}

Based on the above research and analysis, the flow of the improved Canny edge detection algorithm with improved gradient calculation in four directions, and adaptive threshold selection, can be summarized as Fig.1.

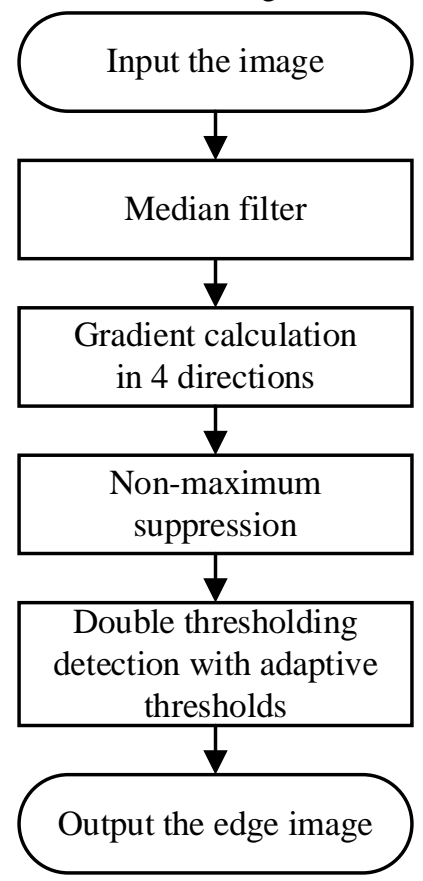

Fig.1. Flow of improved Canny edge detection algorithm

\section{EXPERIMENTAL RESULTS AND DISCUSSIONS}

The proposed improved Canny edge detection algorithm for electronic components images will be verified and compared with the traditional Canny algorithm in this section, both subjectively and objectively. The QFN package image will be taken as an example of SMD electronic components and the lead images will be used as the experimental images. The packages are orderly placed in the specific model tray and then the images are automatically captured and acquired by the industrial camera, as shown in Fig.2. The two algorithms are implemented and experiments are conducted in MATLAB $\mathrm{R} 2020 \mathrm{~b}$ on an industrial computer with $3.6 \mathrm{GHz} \mathrm{CPU}$ and 16GB RAM.

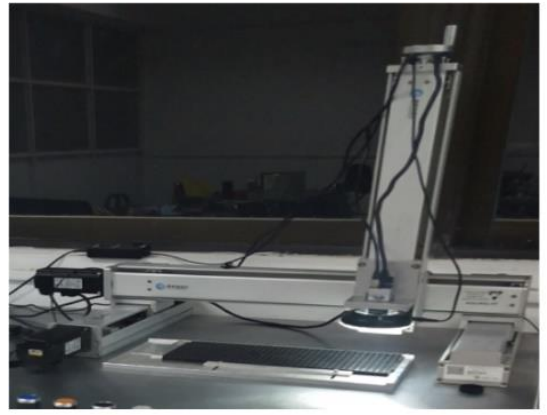

(a)

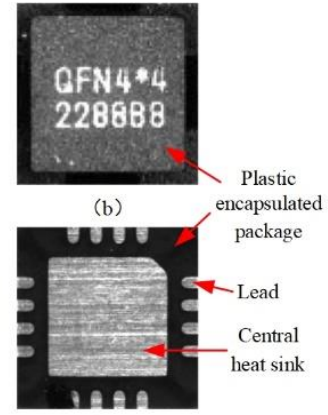

(c)
Fig.2. The experimental platform and objects. a) the image acquisition experimental platform; $b$ ) the package image example of electronic components $(\mathrm{QFN} 4 \times 4)$; c) the lead image example of electronic components (QFN4x4).

The edge location and identification results of both the traditional Canny algorithm and the proposed improved Canny algorithm are shown in Fig.3, Fig.4, and Fig.5, for different QFN lead images, respectively. The standard deviation $\sigma$ of Gaussian function is set to 3 in the traditional Canny algorithm. In order to compare the gradient calculation processes of the two algorithms, the corresponding gradient direction images are also presented in each figure. Visual problem regions of edge results by the traditional algorithm are indicated with red circles.
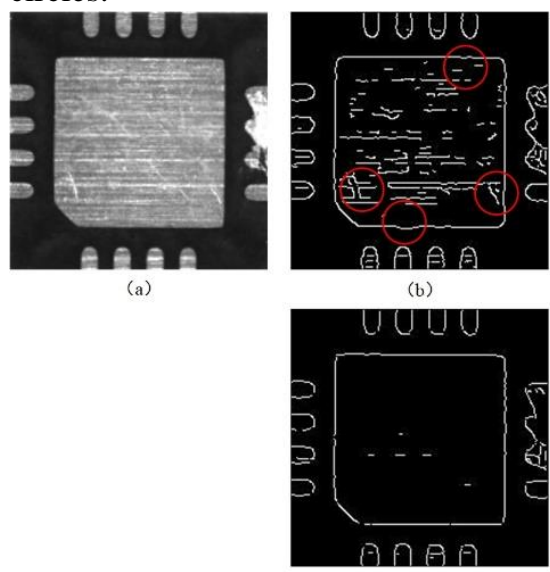

(d)

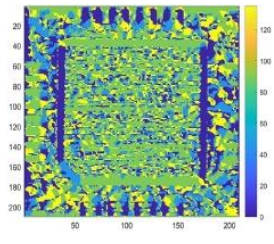

(c)

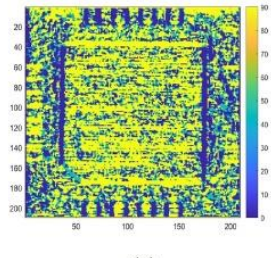

(e)
Fig.3. Edge location and identification results of LEAD1 (QFN4×4). a) the original image of LEAD1 $(210 \times 210)$; b) the edge image by traditional Canny algorithm; c) the gradient direction image by traditional Canny algorithm; d) the edge image by improved Canny algorithm; e) the gradient direction image by improved Canny algorithm. 


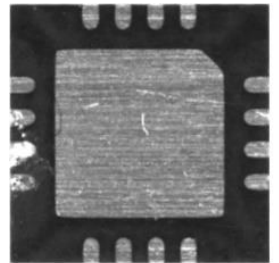

(a)

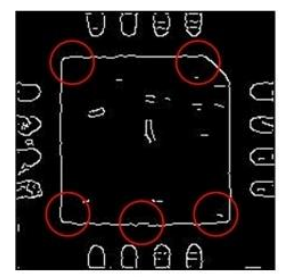

(b)

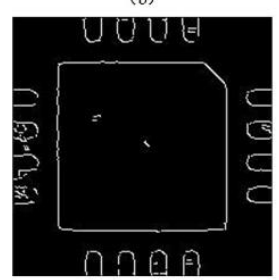

(d)

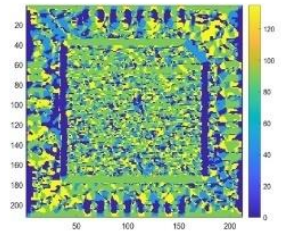

(c)

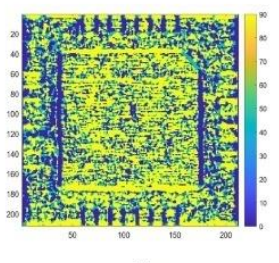

(e)
Fig.4. Edge location and identification results of LEAD2 (QFN4×4). a) the original image of LEAD2 $(210 \times 210)$; b) the edge image by traditional Canny algorithm; c) the gradient direction image by traditional Canny algorithm; d) the edge image by improved Canny algorithm; e) the gradient direction image by improved Canny algorithm.

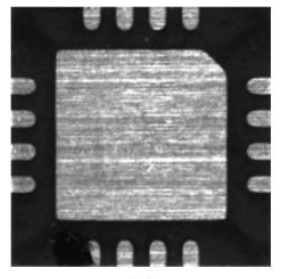

(a)

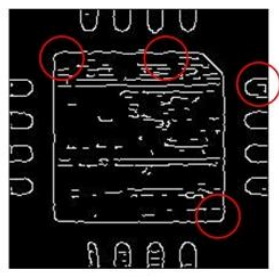

(b)

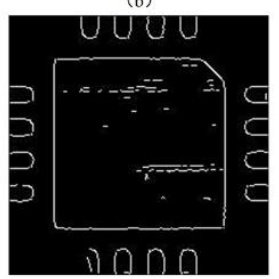

(d)

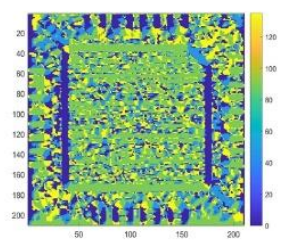

(c)

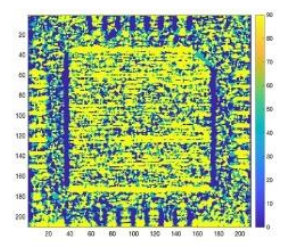

(e)
Fig.5. Edge location and identification results of LEAD3 $(\mathrm{QFN} 4 \times 4)$. a) the original image of LEAD3 $(210 \times 210)$; b) the edge image by traditional Canny algorithm; c) the gradient direction image by traditional Canny algorithm; d) the edge image by improved Canny algorithm; e) the gradient direction image by improved Canny algorithm.

It can be easily found that the defects of shorted lead due to metal connection between leads, lead displacement and foreign material adhesion on leads appear on the right side of Fig.3(a) and left side of Fig.4(a), which are typical but unwanted defects in vision inspection on SMD electronic components. In the process of edge location and identification of leads and central heat sink regions, the occurrence of these defects will change the overall grayscale distribution to some extent and bring challenge to edge detection. Judging from the results of Fig.3(b) and Fig.4(b), the traditional Canny algorithm are influenced by the slight non-linear noise and the texture in the central heat sink region. As a consequence, some false edge points are unwantedly preserved in the central heat sink region, marked with red circles. At the same time, there appears a certain extent of discontinuity along the contour of the heat sink, and part of the linked edges are not aligned and less smooth in both Fig.3(b) and Fig.4(b), compared with the results of the improved Canny algorithm, as shown in Fig.3(d) and Fig.4(d).

Since the texture and contours of both leads and heat sink regions show obvious directionality and distribution regularity, gradient calculation in eight-connectivity and four directions outperforms the original gradient calculation in $2 \times 2$ neighborhood and two directions in non-maximum suppression. The gradient direction distribution of the improved Canny algorithm, as shown in Fig.3(e) and Fig.4(e), is more suitable to the actual regularity than that of the traditional one, as shown in Fig.3(c) and Fig.4(c). Fig.5 shows the edge location and identification results of a QFN lead image with no defect. The evaluation and analysis are similar to the results of Fig. 3 and Fig.4. It is worth noting that some unwanted false edge points are preserved and linked in the lead regions by the traditional Canny algorithm, which will potentially affect the positioning accuracy of the leads, as shown in Fig.5(b).

The double thresholds of the above three experimental images in the process of double thresholding detection, are shown in Table 1, along with the computational time of the two algorithms. The high and low thresholds of the traditional Canny algorithm are selected artificially and empirically, based on the simple statistical property of pixels with fixed parameters.

From Table 1, it can be seen that the double thresholds of the improved Canny algorithm are selected adaptively, depending on the input image, and suitable for the double thresholding detection based on the four-direction gradient information. In terms of computational time, considering the fact that the proposed improved Canny algorithm performs gradient calculation in four directions and the adaptive thresholding selection, the time gap between the traditional and the improved Canny algorithm is slight and acceptable, both of which are no more than 6 milliseconds.

Table 1. Double thresholds and computational time results

\begin{tabular}{ccccccc}
\hline \multirow{2}{*}{ Image } & \multicolumn{2}{c}{$\begin{array}{c}\text { Traditional Canny algorithm } \\
\text { (gradient in 2 directions) }\end{array}$} & \multicolumn{3}{c}{$\begin{array}{c}\text { Improved Canny algorithm } \\
\text { (gradient in 4 directions) }\end{array}$} \\
\cline { 2 - 7 } & High threshod & Low threshod & Time /s & High threshod & Low threshod & Time /s \\
\hline LEAD1 & 0.1875 & 0.0750 & 0.0054 & 0.3765 & 0.1882 & 0.0058 \\
\hline LEAD2 & 0.2344 & 0.0938 & 0.0058 & 0.3725 & 0.1863 & 0.0059 \\
\hline
\end{tabular}




\begin{tabular}{lllllll}
\hline LEAD3 & 0.2344 & 0.0938 & 0.0056 & 0.3843 & 0.1922 & 0.0058 \\
\hline
\end{tabular}

\section{CONCLUSION}

The position identification and defect detection of SMD electronic components with high efficiency play an important role in automatic vision inspection, in which accurate edge location and identification of specific elements in electronic components is the key process. The Canny edge detection algorithm is commonly used to detect the edges of specific elements in the process. The traditional Canny algorithm lacks the adaptability in gradient calculation and double thresholds selection, which may affect the location and identification accuracy of specific elements in electronic components. In this paper, an improved canny edge detection algorithm is proposed. The gradient magnitude is calculated in four directions, i.e., horizontal, vertical, $45^{\circ}$, and $135^{\circ}$. Both the high and low thresholds are adaptively determined based on the grayscale distribution information, to increase the adaptability of edge identification. The experimental results show that the proposed improved Canny algorithm can better locate the true edges of specific elements in electronic components with a reasonable processing speed, compared with the traditional Canny algorithm, and has been successfully applied on practical real-time vision inspection on SMD electronic components.

In the future work, the position of electronic components will be located and marked based on the proposed method. Vision inspection and defect detection on the corresponding electronic circuit products can be performed. Another exploring direction and research emphasis will be the parallel image processing and recognition technique for multiple targets of electronic components in a single frame.

\section{ACKNOWLEDGMENT}

This work was supported in part by the National Natural Science Foundation of China (Grant No. 51905235), and the Natural Science Foundation of Jiangsu Province (Grant No. BK20191037), Changzhou Sci\&Tech Program (Grant No. CJ20190069).

\section{REFERENCES}

[1] Reynolds M R, Campana C, Shetty D. "Design of Machine Vision Systems for Improving Solder Paste Inspection" In ASME 2004 International Mechanical Engineering Congress and Exposition, 2004, pp. 921-929.

[2] Jakati J S, Matad S S. "PCB defect detection based on pattern matching and segmentation algorithm". International Journal of Advanced Research in Computer and Communication Engineering, vol. 3, no. 9, pp.8007-8011, 2014

[3] Singh S, and Bharti M. "Image processing based automatic visual inspection system for PCBs". IOSR Journal Engineering (IOSRJEN), vol. 2, no. 6, pp. 1451-1455, 2012.

[4] Guo F, Guan S. "Research of the machine vision based PCB defect inspection system". In International Conference on Intelligence Science and Information Engineering, 2011, pp. 472-475.

[5] Xiao X, Zhao Y. "Circuit Board Components Positioning and Testing Based on SURF Algorithm". Journal of Tianjin University, vol.47, no.9, pp.765-770, 2014.
[6] Zhou W, Chao Y, Li Y, Li X. "Fast correction of chip image based on improved Harris corner detection". Journal of Electronic Measurement and Instrument, vol.34, no.10, pp.74-81, 2020.

[7] Chen G, Li H, Fang X. "Research of the Vision Positioning System of Surface Mounting Machine Based on Phase Congruence and Hough Circle Transform". Science Technology \& Engineering, vol.27, no.11, pp.59-63, 2015.

[8] Jiang T, Wang A, Wang S, Shao M. "An Error Correction Method for PCB Based on Machine Vision". Journal of Shanghai Jiaotong University, vol.24, no.6, pp.945-949, 2005.

[9] Raihan F, Ce W. "PCB defect detection USING OPENCV with image subtraction method". In International Conference on Information Management and Technology, 2017, pp. 204-209.

[10] Chao Y, Dai M, Chen K, Chen P, Zhang Z. "A novel gravitational search algorithm for multilevel image segmentation and its application on semiconductor packages vision inspection". Optik - International Journal for Light and Electron Optics, vol 127, n 14, pp.5770-5782, 2016.

[11] Ganavi V M, Rao M. "Printed Circuit Board Assembly defects detection using image processing techniques". International Journal of Engineering Science and Computing, vol. 6, no. 6, pp.7824-7829, 2016.

[12] Borthakur M, Latne A, Kulkarni P. "A comparative study of automated PCB defect detection algorithms and to propose an optimal approach to improve the technique". International Journal of Computer Applications, vol. 114, no. 6, pp.27-33, 2015.

[13] Lee D H, Chen P Y, Yang F J, et al. "High-Efficient Low-Cost VLSI Implementation for Canny Edge Detection". Journal of Information Science \& Engineering, Vol. 36, no. 3, 2020.

[14] Sridevi S, Muralidharan G, Kumar C N. "Online inspection of printed circuit board using machine vision". International Journal of Innovative Research in Science, Engineering and Technology, vol. 3, no. 3, pp.230-238, 2014.

[15] Canny J. "A computational approach to edge detection". IEEE Transactions on Pattern Analysis and Machine Intelligence, vol. 8, no. 6, pp.679-698, 1986.

[16] Chao Y, Dai M, Zhang Z. "Fast image acquisition and magnification of QFN plastic encapsulated packages". In International Conference on Mechatronics and Machine Vision in Practice, 2016.

\section{Creative Commons Attribution License 4.0 (Attribution 4.0 International, CC BY 4.0)}

This article is published under the terms of the Creative Commons Attribution License 4.0 https://creativecommons.org/licenses/by/4.0/deed.en_US 